\title{
A Markov Perspective on C-V2X Mode 4
}

\author{
Geeth P. Wijesiri N.B.A \\ Center for Wireless Communications, \\ University of Oulu, Finland \\ University of Moratuwa, Sri Lanka \\ University of Ruhuna, Sri Lanka \\ geeth@eie.ruh.ac.lk
}

\author{
Jussi Haapola \\ Center for Wireless Communications, \\ University of Oulu, Finland \\ jussi.haapola@oulu.fi
}

\author{
Tharaka Samarasinghe \\ Dept. of Electronic and Telecommunication \\ Engineering, University of Moratuwa, \\ Sri Lanka \\ University of Melbourne, Australia \\ tharakas@uom.lk
}

\begin{abstract}
This paper proposes a multi-dimensional Markov model to evaluate the medium access control (MAC) layer performance of 3GPP cellular vehicle to everything (V2X) Mode 4. The Mode 4 specifications enable quality of service guarantees in a decentralized manner, without the connectivity of a cellular base station, and therefore, ideal for V2X messaging in intelligent transportation systems (ITS). To this end, periodic cooperative aware messages (CAMs) and event triggered decentralized environmental notification messages (DENMs) are considered in the paper. The discrete time Markov model consists of a node model, a queue model, and traffic generators for CAM and DENM packets that are dependent and solved in closed form and iteratively. The model is applied to a highway scenario to provide insights on the average delay, the collision probability, and the channel utilization in Mode 4. The results show that Mode 4 has been partially over-specified for ITS applications and able to serve CAMs and DENMs conveniently. However, there exists locally optimal combinations of CAM and DENM intervals that can lead to lower average delay.
\end{abstract}

Index Terms-3GPP, cooperative aware messages, C-V2X, decentralized environmental notification messages, discrete time Markov chain, intelligent transport systems

\section{INTRODUCTION}

Vehicular networks will primarily depend on vehicle-tovehicle $(\mathrm{V} 2 \mathrm{~V})$ communications to enable an active safety environment. $\mathrm{V} 2 \mathrm{~V}$ communications mainly consists of an exchange of small broadcast packets that have critical latency and reliability requirements. It has gained considerable research interest, with a focus of developing vehicular networks using the IEEE $802.11 \mathrm{p} /$ dedicated short-range communication (DSRC) as an enabling technology. However, as emphasized in [1], the $802.11 \mathrm{p}$ medium access control (MAC) protocol has issues in guaranteeing quality-of-service (QoS) levels, and in ensuring the networks' scalability. As an alternative to $802.11 \mathrm{p}$, the third generation partnership project (3GPP) published the first version of the Release 14 [2] standard including support for $\mathrm{V} 2 \mathrm{~V}$ communications using long-term evolution (LTE) sidelink communications (a.k.a. LTE-V, LTEV2X, LTE-V2V, or Cellular-V2X (C-V2X)).

LTE sidelink was introduced for the public safety deviceto-device (D2D) communications in Release 12 as Mode 1 and Mode 2. Release 14 introduces Mode 3 and Mode 4, specifically designed for $\mathrm{V} 2 \mathrm{~V}$ communications [2], [4]. In Mode 3, two vehicles directly communicate with each other although the selection and management of the resources are carried out by the cellular infrastructure. In Mode 4 [4], [5], vehicles autonomously select and manage their resources without any cellular infrastructure support. Mode 4 is highly applicable for V2V based safety applications, which cannot depend on the availability of mobile coverage.

An essential novel feature introduced in Mode 4 is the distributed scheduling protocol that enables vehicles to autonomously select their radio resources without any infrastructure. The protocol is referred to as sensing-based semipersistent scheduling (SPS). Its objective is to improve the reliability of $\mathrm{V} 2 \mathrm{~V}$ communications without overloading the channel with retransmissions. Mode 4 manages the periodic nature of cooperative aware messages (CAMs) and Poisson nature of decentralized environment notification messages (DENMs) by utilizing semi-persistent scheduling of resources. Vehicles sense the previous transmissions of other vehicles to estimate free ones, hence avoiding packet collisions. The ability of Mode 4 to guarantee reliable V2V communication strongly depends on the satisfactory operation of the sensingbased SPS algorithm.

The first analytical model for C-V2X was proposed in [6]. The paper introduced a set of novel techniques to study the communication performance of C-V2X Mode 4. In particular, the paper presented analytical results for the average packet delivery ratio (PDR) as a function of the distance between the transmitter and the receiver, and for four different types of transmission errors that can be encountered in Mode 4. The models were validated for different transmission parameters and traffic densities, by using a simulator implemented over the Veins simulation platform [7].

The main contribution of this paper is analytical modeling of the MAC layer performance of C-V2X Mode 4 using discrete time Markov chains (DTMC), which makes it different to [6]. The complete Markov model consists of a state machine and a queue model to represent the SPS mechanism and the device level packet queue, respectively. Moreover, the packet queue contains hidden Markov model traffic generators for CAM and DENM. These multiple parallel Markov processes are solved iteratively. The steady-state probabilities are used to calculate parameters of C-V2X Mode 4 such as successful transmission probability of a vehicle, average delay, collision probability and channel utilization.

The remainder of this paper is organized as follows. The developed Markov models and steady-state solutions are presented in Sections II and III, respectively. The numerical results are presented in Section IV and the paper is concluded in Section V. 


\section{MARKov Model}

DTMC based model of C-V2X Mode 4 communication consists of four DTMCs that are in parallel. The first DTMC models the C-V2X Mode 4 node operation with the SPS algorithm for radio resource selection, denoted as the state machine. The other three DTMCs are the CAM and the DENM packet generators, and the device level packet queue. All the DTMCs are ergodic, i.e. they are aperiodic and positive recurrent, hence a steady-state distribution exists.

\section{A. Semi-persistant scheduling algorithm}

The main purpose of the SPS algorithm [5] is enabling the selection of radio resources for the vehicle without the assistance of an eNodeB. Based on the SPS algorithm, the following three steps are followed by each vehicle to select the suitable candidate for resource reservation.

Step 1: Within the selection window, which is a time window that initiates with the packet generation, vehicle $v$ identifies all possible candidate single-subframe $(1 \mathrm{~ms})$ resources (CSRs) that can be reserved. CSRs are groups of adjacent sub-channels within a given subframe that are large enough to fit in the sidelink control information (SCI) and the transport block (TB), which will be transmitted. The length of the selection window is defined in the standard as the maximum latency [4], and a CSR should be selected within this duration.

Step 2: $v$ creates list $L_{1}$ that consists of CSRs it can reserve, based on information it receives in the previous 1000 subframes (sensing window). $L_{1}$ includes all the CSRs in the selection window except those that satisfy the following conditions.

1) CSRs used by vehicle $v$ during the sensing window. This is due to $v$ not being able to sense these CSRs during its half duplex transmissions.

2) CSRs that have a received signal strength indicator (RSSI) value above a threshold level $\left(l_{t h}\right)$, and are being used by other vehicles at the time $v$ tries to utilize them.

If $L_{1}$ contains more than $20 \%$ of the total CSRs in the selection window, the system moves to Step 3. Otherwise, $l_{t h}$ is increased by $3 \mathrm{~dB}$ and Step 2 is repeated.

Step 3: From $L_{1}, v$ filters out the CSRs that experience the highest average RSSI. For each CSR, the averaging is done over the previous 10 subframes. Out of these CSRs, the CSRs with the lowest average RSSI values are added to a new list $L_{2}$ such that the size of $L_{2}$ amounts to $20 \%$ of the total CSRs in the selection window. $v$ randomly and uniformly selects a CSR in $L_{2}$ and reserves it for the next $R C$ transmissions, where $R C$ denotes the value of the reselection counter. $R C$ is decremented by 1 for each transmission of a packet, and after $R C=1$, new CSRs should be selected and reserved with probability $\left(1-P_{r k}\right)$, where $P_{r k} \in[0,0.8]$.

\section{B. State machine}

Fig.1 illustrates the DTMC model for C-V2X Mode 4 operation of a vehicle. Transition probabilities and states in the model are as follows. State Idle represents the state with

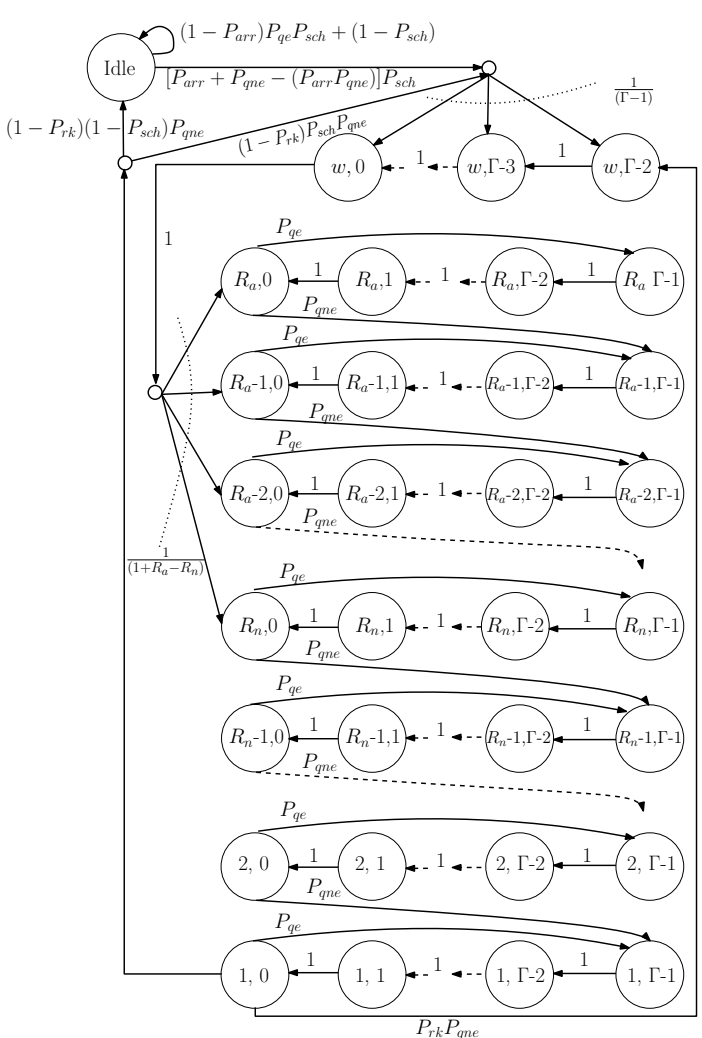

Figure 1. DTMC model for the state machine.

no packets to transmit, or no CSRs to transmit. We assume a vehicle may not have packets to transmit during certain periods (unsaturated conditions). The waiting states between packet arrival and the selection of a radio resource to transmit are represented using $w$. The transition probabilities are defined as $P_{\text {sch }}=\operatorname{Pr}\{$ Node finds a suitable CSR (step 1-3) $\}, P_{\text {qne }}=$ $\operatorname{Pr}\{$ Queue not empty $\}, P_{q e}=\operatorname{Pr}\{$ Queue empty $\}$, and $P_{\text {arr }}$ $=\operatorname{Pr}\{$ New packet arrival $\mid$ queue is empty $\}$. The lower and upper limits of the reselection counter's selection window are denoted by $R_{n}$ and $R_{a}$, respectively. The $\Gamma$ denotes the size of the selection window in ms. According to the standard [2], there are three selection window sizes, with their respective reselection counter selection ranges. To this end, the standard includes $\Gamma=100 \mathrm{~ms}$ with $R C \in[5,15], \Gamma=50 \mathrm{~ms}$ with $R C \in[10,30]$ and $\Gamma=20 \mathrm{~ms}$ with $R C \in[25,75]$. In this paper, we consider $\Gamma=100 \mathrm{~ms}$ with $R C \in[5,15]$, and the remaining two scenarios will be considered in our future work. If a new packet arrives when $R C=0$, the SPS algorithm has to allocate a radio resource for the transmission of this packet. The time allocated to the SPS algorithm for this activity is $(\Gamma-1) \mathrm{ms}$. Hence, the waiting time duration before the transmission of a packet is represented by $\Gamma-1$ equiprobable states, $(w, 0)$ to $(w, \Gamma-2)$.

At each $(i, 0)$ state where $i \in\{1, \ldots, 15\}$, the vehicle transmits a packet, and let $\pi_{i, 0}$ denote the steady-state probability of the respective state. The probability of successful transmission of a packet is given by $P_{s}=\sum_{i=1}^{R_{a}} \pi_{i, 0}$. After the waiting time is over, $v$ selects a value randomly and uniformly from 


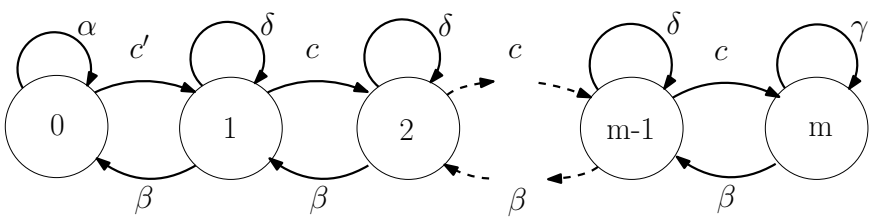

Figure 2. DTMC model for the packet queue of a vehicle.

the set of $\left(1+R_{a}-R_{n}\right) R C$ values, If the queue is empty, the system stays in the $R C$ value for $\Gamma \mathrm{ms}$, or otherwise, moves to $(R C-1, \Gamma-1)$ state. If the queue is not empty when the arrives at the $(1,0)$ state, the vehicle has to select new radio resources. To this end, the vehicle will select a new radio resource with probability $1-P_{r k}$, and use the same radio resource as in the previous transmission with probability $P_{r k}$.

\section{Queue model}

The queue model represents the packet arrival queue of a vehicle for two types of packets, CAM and DENM. CAM queue is modeled using a fixed inter-arrival time model, where the inter-arrival time is set according to the standard. The DENM queue is modeled using a Poisson arrival model since DENM are event-triggered messages. Fig. 2 illustrates the DTMC queue model, where the maximum size of the queue is considered to be $m$. Each state of the queue model is explained by the traffic generator DTMC model shown in Fig. 3. In Fig. 3, the transition probabilities associated with the CAM and DENM packet generation are represented by the blue doted lines and the red dash lines, respectively. The transition probabilities common to both processes are represented using the solid black lines and $x \in\{t, z\}$. We consider $x=t$ for the CAM generator, and it represents the inter-arrival time between CAM packets. We consider $x=z$ for the DENM generator, and it represents the repetition frequency of DENM packets. Furthermore, in the DENM model, $k$ denotes the average number of repetitions and $\lambda$ denotes the DEMN packet arrival rate (pac/s). For DENM packets, by using the Poisson arrival assumption, the probability of at least one packet arriving in interval $T$ is given by $1-e^{-\lambda T}$. Moreover, in the DENM generator, an additional idle state can be observed compared to the CAM generator. In the state notation, $e$ and $c$ denote the previous and the current state, respectively. The transition probabilities of the queue model are derived from steady-state probabilities of the generator models.

Let $\pi_{i, j}^{C A M}$ and $\pi_{i, j}^{D E N M}$ denote the steady-state probabilities of the $(i, j)$ state of the CAM and DENM generators, respectively. For the CAM generator, the steady-state probabilities are given by $\pi_{e, i}^{C A M}=P_{s}\left(\pi_{e, 0}^{C A M}+\sum_{j=i+1}^{t-1} \pi_{c, j}^{C A M}\right) \forall i \in[1, t-2]$, $\pi_{c, i}^{C A M}=\pi_{e, 0}^{C A M} \frac{\left(1-P_{s}\right)^{t-i}}{\left[1-\left(1-P_{s}\right)^{t-1}\right]} \forall i \in[0, t-1], \pi_{e, t-1}^{C A M}=\pi_{e, 0} P_{s}$, and

$$
\begin{aligned}
\pi_{e, 0}^{C A M}=\left[1+(t-1) P_{s}+P_{s}\right. & \sum_{i=1}^{t-2} \sum_{j=i+1}^{t-1} \frac{\left(1-P_{s}\right)^{(t-j)}}{\left[1-\left(1-P_{s}\right)^{(t-1)}\right]} \\
& \left.\left.+\sum_{i=0}^{t-1} \frac{\left(1-P_{s}\right)^{(t-i)}}{\left[1-\left(1-P_{s}\right)^{(t-1)}\right]}\right]\right]^{-1}
\end{aligned}
$$

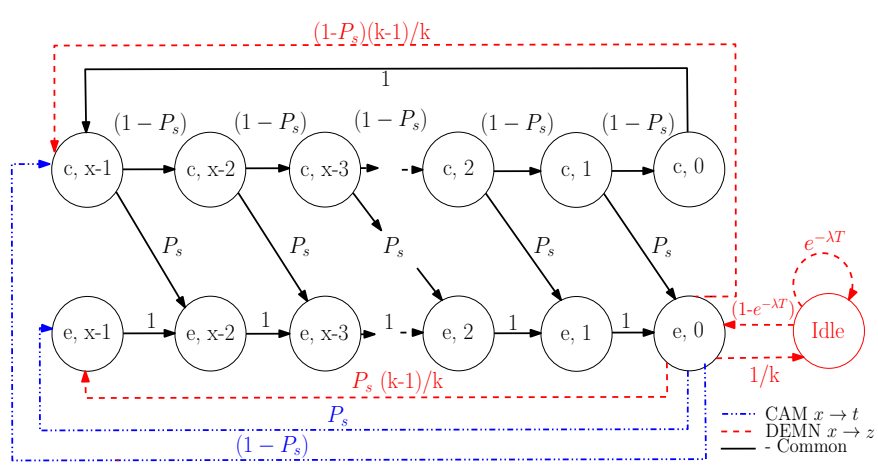

Figure 3. DTMC model for CAM \& DENM packet generation.

Similarly, the steady-state probabilities of the DENM generator are given by $\pi_{e, i}^{D E N M}=P_{s}\left[\frac{(k-1)}{k} \pi_{e, 0}^{D E N M}+\sum_{j=i+1}^{z-1} \pi_{c, j}^{D E N M}\right] \forall i \in$ $[1, z-2], \pi_{c, i}^{D E N M}=\pi_{e, 0}^{D E N M} \frac{(k-1)}{k} \frac{\left(1-P_{s}\right)^{z-i}{ }^{j=i+1}}{\left[1-\left(1-P_{s}\right)^{z-1}\right]} \forall i \in[0, z-1]$, $\pi_{e, z-1}^{D E N M}=\pi_{e, 0}^{D E N M} \frac{(k-1)}{k} P_{s}, \pi_{\text {Idle }}=\frac{1}{\left(1-e^{-\lambda T}\right) k} \pi_{e, 0}$ and

$$
\begin{aligned}
\pi_{e, 0}^{D E N M}=\left[1+(z-1) P_{s}\right. & \frac{(k-1)}{k}+P_{s} \frac{(k-1)}{k} \sum_{i=1}^{z-2} \sum_{j=i+1}^{z-1} \frac{\left(1-P_{s}\right)^{(z-j)}}{\left[1-\left(1-P_{s}\right)^{(z-1)}\right]} \\
& \left.+\frac{(k-1)}{k} \sum_{i=0}^{z-1} \frac{\left(1-P_{s}\right)^{(z-i)}}{\left[1-\left(1-P_{s}\right)^{(z-1)}\right]}+\frac{1}{k\left(1-e^{-\lambda T}\right)}\right] \cdot \cdot^{-1}
\end{aligned}
$$

In the queue model, the CAM and the DENM transition probabilities can be derived using the steady-state probabilities above. Let $c_{i}^{\prime}, \beta_{i}$, and $c_{i}$, where $i \in\{\mathrm{CAM}, \mathrm{DENM}\}$, denote the transition probabilities of the CAM generator and the DENM generator, respectively. Then, we have $c_{C A M}^{\prime}=\pi_{e, 0}\left(1-P_{s}\right)+\pi_{c, 0}, \quad c_{C A M}=\pi_{c, 0}, \quad \beta_{C A M}=$ $\sum_{i=1}^{t-1} \pi_{c, i} P_{s}, c_{D E N M}^{\prime}=\pi_{e, 0} \frac{(k-1)}{k}\left(1-P_{s}\right)+\left(1-e^{-\lambda T}\right) \pi_{I}+$ $\pi_{c, 0}, \quad c_{D E N M}=\pi_{c, 0}+\left(1-e^{-\lambda T}\right) \pi_{I}$ and $\beta_{D E N M}=$ $\sum_{i=1}^{t-1} \pi_{c, i} P_{s}$. The resultant transition probabilities of the queue model are given by $c^{\prime}=\left(c_{C A M}^{\prime}+c_{D E N M}^{\prime}\right)-$ $\left(c_{C A M}^{\prime} \times c_{D E N M}^{\prime}\right), c=\left(c_{C A M}+c_{D E N M}\right)-\left(c_{C A M} \times\right.$ $\left.c_{D E N M}\right)$ and $\beta=\left(\beta_{C A M}+\beta_{D E N M}\right)-\left(\beta_{C A M} \times \beta_{D E N M}\right)$.

Hence, the steady-sate probability of state 0 of the queue model is given by

$$
\pi_{0}=\left[1+c^{\prime}\left(\frac{1-\beta^{-m} c^{m}}{\beta-c}\right)\right],{ }^{-1}
$$

and the steady-state probability of state $i$ where $i \in[1, m]$, is given by

$$
\pi_{i}=\frac{c^{\prime} c^{i-1}}{\beta^{i}} \pi_{0} .
$$

\section{Steady-State Solution}

Steady-state solutions of the state machine and the queue models are calculated in parallel. Probabilities $P_{s}, P_{s c h}, P_{\text {arr }}$, and $P_{r k}$ are initialized and $P_{s}$ and $P_{q e}$ are recomputed iteratively based on the steady-state probabilities, until the probability values stop varying significantly. A value for $\lambda$ is selected based on the interarrival time of the DENM packets. 


\section{A. Interpreting $P_{\text {sch }}$}

We consider $A$ CSRs in the selection window and that $X$ CSRs are excluded according to the SPS algorithm, such that there are $A-X$ CSRs in $L_{1}$. Let $N$ be the number of vehicles in the area of influence of $v$. The value of $X$ will depend on $\tilde{X}$, which denotes the number of times we encounter $R C=1$ in a given sensing window. Let $B$ denote the ratio between the sizes of the sensing window and the selection window. Since $B \leq 2 R_{n}$ according to the standard, we have $\tilde{X} \in$ $\{0,1,2\}$. Let $P_{\tilde{X}=i}=\operatorname{Pr}\{\tilde{X}=i\}$, and let $C_{\tilde{X}=i}$ denote the total number of realizations (count) of the sensing window that satisfy $\tilde{X}=i$, for $i \in\{0,1,2\}$. Let $P_{s r}=P_{\tilde{X}=0}+P_{r k}(1-$ $\left.P_{\tilde{X}=0}\right)$, which gives us the probability of maintaining the same CSR throughout the sensing window. Then, we can show that $X=(N-1)\left(P_{s r}+P_{\tilde{X}=1}+P_{\tilde{X}=2}\right)+\left(P_{s r}+2 P_{\tilde{X}=1}+3 P_{\tilde{X}=2}\right)$.

Let $R C_{F} \in\left[R_{a}, 1\right]$ be the first $\mathrm{RC}$ value of the sensing window. The set of possible $R C_{F}$ values can be represented as $\left\{S_{1}, S_{2}, S_{3}\right\}$, using three subsets, where $S_{1}=\left\{R_{a}, R_{a}-\right.$ $1, \ldots, B+1\}, S_{2}=\left\{B, B-1, \ldots, B-R_{n}+1\right\}$, and $S_{3}=\{B-$ $\left.R_{n}, B-R_{n}-1, \ldots, 1\right\}$. When $R C_{F} \in S_{1}$, it is not hard to see that $\tilde{X}=0$, and we have $C_{\tilde{X}=0}=R_{a}-B$. If $R C_{F} \in S_{2}$, we encounter $\tilde{X}=1$, and we can show the count given $R C_{F} \in S_{2}$ be $C_{\tilde{X}=1, S_{2}}=\left(R_{a}-R_{n}+1\right)\left(R_{n}-1\right)+1$. Finally, if $R C_{F} \in S_{3}, \tilde{X}$ can either be 1 or 2 , and their counts can be shown to be $C_{\tilde{X}=1, S_{3}}=(1 / 2)\left(B^{2}+B-R_{n}\left(R_{n}+1\right)\right)$ and $C_{\tilde{X}=2}=(1 / 2)\left(B-R_{n}\right)\left[R_{a}\left(B-R_{n}-1\right)-B R_{n}+B+R_{n}^{2}+1\right]$, respectively. We have $C_{\tilde{X}=1}=C_{\tilde{X}=1, S_{2}}+C_{\tilde{X}=1, S_{3}}$, and $P_{\tilde{X}=i}=$ $C_{\tilde{X}=i} / \sum_{j=0}^{2} C_{\tilde{X}=i}$ for $i \in\{0,1,2\}$.

Next, we will further evaluate $X$ with reference to the standard. According to the 3GPP C-V2X standard [4], singlecarrier frequency-division multiple access (SC-FDMA) is considered for the uplink, using a $10 \mathrm{MHz}$ channel. 50 Resource Blocks (RB) are allocated for this bandwidth per each slot (half sub-frame), and hence, one sub-frame contains 100 RBs. A CSR requires at least 4 RBs to transmit a 100 byte payload, using 64 QAM modulation. Therefore, each $1 \mathrm{~ms}$ sub-frame can hold up to $25 \mathrm{CSRs}$, and hence the $100 \mathrm{~ms}$ selection window can hold up to 2500 CSRs. This means, $L_{1}$ should include at least 500 CSRs to satisfy the requirement in Step 2 of the SPS algorithm, or $X$ should be less than 2000 CSRs. If we consider $X>2000$, from (3) we have

$$
N>\frac{2000-P_{\tilde{X}=1}-2 P_{\tilde{X}=2}}{P_{\tilde{X}=0}+\left(\max \left(P_{r k}\right)\right)\left(1-P_{\tilde{X}=0}\right)+P_{\tilde{X}=1}+P_{\tilde{X}=2}} .
$$

By substituting values for $P_{\tilde{X}=1}, P_{\tilde{X}=2}$ and $\max \left(P_{r k}\right)=0.8$, we get $N>1123$. This is a very unlikely condition in a highway scenario. Therefore, without a loss of generality, we assume $P_{s c h}$ to be 1 in our analysis.

\section{B. Steady-state solutions}

The steady-state equations of the state machine in Fig. 1 are used to derive its steady-state distribution, which is presented next. To this end,

States $(w, i)$ :

$$
\begin{aligned}
\pi_{w, i}= & a\left(\frac{\Gamma-1-i}{\Gamma-1}\right) \pi_{I}+\left[\frac{(\Gamma-1-i)}{(\Gamma-1)} P_{q n e}\left(1-P_{r k}\right) P_{s c h}\right. \\
& \left.+P_{q n e} P_{r k}\right] \pi_{1,0}, \forall i \in[0, \Gamma-2],
\end{aligned}
$$

where $a=\left(P_{\text {arr }}+P_{q n e}-P_{\text {arr }} P_{q n e}\right) P_{\text {sch }}$.

States $(i, j)$ :

$$
\begin{array}{r}
\pi_{i, j}=\left(\frac{R_{a}-i+1}{P_{q n e}}\right) \frac{1}{P_{q n e}\left(1+R_{a}-R_{n}\right)} \pi_{w, 0}, \\
\forall i \in\left[R_{n}, R_{a}\right], \forall j \in[1, \Gamma-1] . \\
\pi_{i, j}=\frac{\pi_{w, 0}}{P_{q n e}}, \forall i \in\left[1, R_{n}-1\right], \forall j \in[0, \Gamma-1] .
\end{array}
$$

States $(i, 0)$

$$
\pi_{i, 0}=\frac{\left(R_{a}-i+1\right)}{P_{q n e}\left(1+R_{a}-R_{n}\right)} \pi_{w, 0}, \forall i \in\left[R_{n}, R_{a}\right] .
$$

State Idle:

$$
\begin{array}{r}
\pi_{\text {Idle }}=\frac{\left[\left(1-P_{r k}\right)\left(\frac{1}{P_{s c h}}-1\right)\right]}{\left[P_{\text {arr }}+P_{\text {qne }}\left(1-P_{\text {arr }}\right)\right]} \pi_{w, 0}=b \pi_{w, 0}, \\
\text { where } b=\frac{\left[\left(1-P_{r k}\right)\left(\frac{1}{P_{s c h}}-1\right)\right]}{\left[P_{\text {arr }}+P_{\text {qne }}\left(1-P_{\text {arr }}\right)\right]}
\end{array}
$$

Since the sum of probabilities is one, we have

$$
\begin{array}{r}
\pi_{w, 0}=\left[1-\Gamma+b+\left(\frac{\Gamma-2}{2}\right)\left[a b+2 P_{r k}+\left(1-P_{r k}\right)\right.\right. \\
\left.\left.P_{s c h}\right]+\frac{\Gamma\left(R_{a}+R_{n}\right)}{2 P_{\text {qne }}}\right]^{-1} .
\end{array}
$$

Markov models are solved in parallel until repetitive iterations do not significantly change the steady-state probabilities. The following important probabilities, $P_{s}=\sum_{j=1}^{R_{a}} \pi_{j, 0}$ and $P_{q e}=\pi_{0}$ from (1) are determined from the steady-state solutions of the models.

\section{Numerical Results}

In this section, we apply the model for a highway scenario, and provide insights on key performance indicators through simulations.

\section{A. Instantiation of CAM and DENM in highway}

We consider a highway with four parallel lanes in each direction. We assume that the vehicles move at a constant speed of $100 \mathrm{~km} / \mathrm{h}$ on the highway, and the average intervehicle distance is $50 \mathrm{~m}$. We assume that only CAM and DENM are utilized for V2V communication [9], [10], where their reference packet formats are specified by the European telecommunications standards institute (ETSI) [8]. CAM, which are keep alive messages, are periodically broadcast by each vehicle to provide information of presence, position, temperature, and primary status. DENM are event-triggered messages, which are broadcast to alert the road users of a hazardous event. Both CAM and DENM are delivered to vehicles in the geographical region of influence by the target vehicle. 


\section{B. Instantiation of the Markov model}

To initialize the DTMC model for the state machine, we use $P_{s}=0.1, P_{r k}=0.4$ and $P_{s c h}=1$. A maximum size of 10 packets $(m=10)$ is considered in the DTMC model for the packet queue. $R_{n}=5, R_{a}=15$, and $\Gamma=100$ are set according to the standard [5]. Then, probability values for $P_{s}$ and $P_{q n e}$ are obtained from the steady-state distributions of the state machine and the queue model. We consider the CAM packet inter-arrival time to be between $100 \mathrm{~ms}$ and 1 $\mathrm{s}$, according to the specifications [8]. The inter-arrival time of the event triggered DENM is modeled by a Poisson process, and the average repetition frequency of DENM packets is considered to be $z=100,200$ and $300 \mathrm{~ms}$, and the average number of repetitions of packets to be between $k=1$ and 9. The packet arrival rate $\lambda$ is considered to be $1 \mathrm{pac} / \mathrm{s}, 0.2$ $\mathrm{pac} / \mathrm{s}$, and $0.011 \mathrm{pac} / \mathrm{s}$. The iterative solution for $P_{s}$ converges to 0.0102 .

\section{Average delay $d_{\text {ave }}$}

The average delay between the generation and the transmission of a packet is given by

$$
d_{\text {ave }}=\frac{\sum_{i=1}^{m} \frac{2 i-1}{2 P_{s}} \pi_{i}}{1-P_{q e}} .
$$

The delay value captures the queuing delay and the access delay, which are introduced in the queue model and the state machine, respectively.

1) The behavior of $d_{\text {ave }}$ with the inter-arrival time of CAMs: The behavior of $d_{\text {ave }}$ with the inter-arrival time of CAMs is illustrated in Fig. 4 for different parameter combinations of the DENM model. The main observation is that the delay reduces with $t$ at the start. We can observe the highest average delay when $z=100$ and the lowest when $z=300$, and the average delay decreasing when the repetition frequency of DENM packets is increased. When $z=100$, we can observe the average delay increasing when $k$ is increased from 1 to 9 . On the other hand, when $z=200$ and $z=300$, we can observe the average delay decreasing when $k$ is increased from 1 to 9. This phenomenon can be reasoned out as follows. When $z=100$, the service rate is nearly equal to the packet repetition frequency. This results in more CAM and DENM packets in the queue, leading to higher queuing delays. However, when $z=200$ and $z=300$, the service rate is higher than the repetition frequency of DENM packets. In such a scenario, increasing the average number of repetitions results in the system encountering the random waiting time, which has an average delay of $50 \mathrm{~ms}$, more frequently compared to waiting through the whole resource reservation interval (RRI), which is of $100 \mathrm{~ms}$. Thus, we can observe the average delay decreasing.

Another interesting observation in the behavior of the average delay is the existence of a local optimal point. For an example, when $z=100, k=9$ and $\lambda=0.2 \mathrm{pac} / \mathrm{s}$, the lowest average delay can be observed at $t=300 \mathrm{~ms}$. Moreover, we can observe the average delay increasing when the DENM packet arrival rate is increased from $0.011 \mathrm{pac} / \mathrm{s}$ to $1 \mathrm{pac} / \mathrm{s}$.

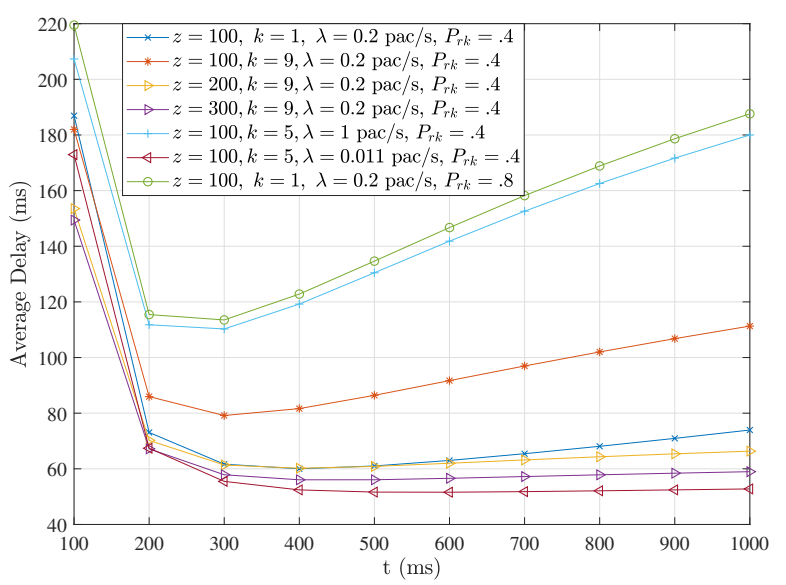

Figure 4. The behavior of the average delay with the inter-arrival time of CAMs $(N=50)$.

2) The behavior of $d_{\text {ave }}$ with $P_{r k}$ : We can also observe the average delay increasing with $P_{r k}$ in Fig. 4. High values of $P_{r k}$ curtails the vehicle from choosing new radio resources for the transmission. When $P_{r k}$ is low, a vehicle receives more opportunities to encounter the waiting interval (average duration of $50 \mathrm{~ms}$ ), compared to the longer RRI intervals (duration of $100 \mathrm{~ms}$ ). Thus, high $P_{r k}$ values lead to higher average delays.

\section{Probability of collision $P_{\text {col }}$}

Even though the SPS algorithm attempts to minimize packet collisions between vehicles at transmission by considering the radio resource utilization of vehicles during the $1000 \mathrm{~ms}$ sensing window, there still remains a possibility for collisions. To this end, a schedule collision can occur when a vehicle selects a new radio resource for transmissions. Such a collision can occur during a vulnerable period of $100 \mathrm{~ms}$, which is equal to the length of the selection window, as shown in Fig. 5. For the calculation of the collision probability $P_{c o l}$, first, the cycle time of the state $(1,0)$ must be calculated. After state $(1,0)$, the vehicle decides to select new radio resources or to use the existing resource, based on the value of $P_{r k}$. As the steady-state probability of $\pi_{1,0}$ is already known from the Markov model, the cycle time of state $(1,0)$ can be calculated by $u=1 / \pi_{1,0}$. Then, for a $100 \mathrm{~ms}$ selection window, the probability of state $(1,0)$ not being visited in the selection

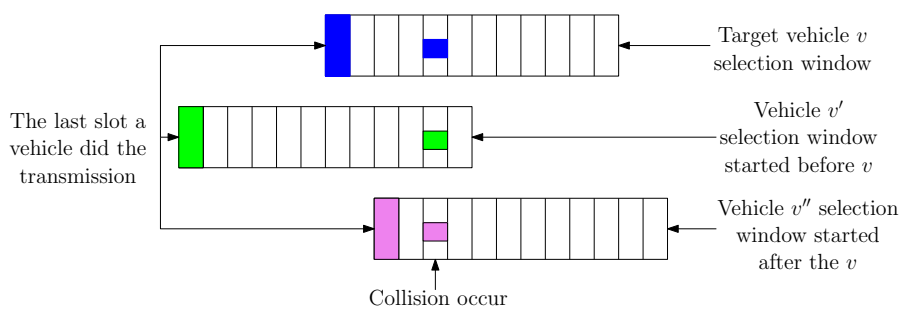

Figure 5. The possibility of a collision in the vulnerable period. 


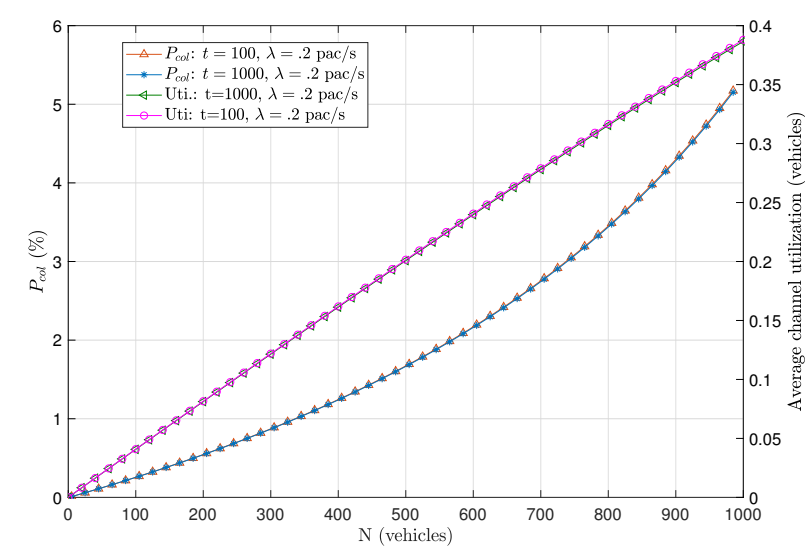

Figure 6. $P_{c o l}$ and the average channel utilization with $\mathrm{N}(k=5, z=100$, and $\left.P_{r k}=.4\right)$.

window is given by $q=\prod_{i=1}^{99}\left(1-\frac{1}{u-i}\right)$. Hence, for $N$ vehicles

$$
P_{\text {col }}=1-\left[1-\left\{(1-q)\left(1-P_{r k}\right) \frac{1}{(A-X)}\right\}\right]^{N-1} \text {. }
$$

1) The behavior of $P_{\text {col }}$ with $N$ : We can observe $P_{c o l}$ increasing with $N$ as illustrated in Fig. 6. We can also observe that this behavior does not change significantly for different CAMs and DENMs arrival rates (the absolute difference is less than $1 \%$ ).

2) The behavior of $P_{c o l}$ with $P_{r k}$ : We can observe $P_{c o l}$ decreasing with $P_{r k}$. Similar to above, the behavior does not change significantly for different CAMs and DENMs arrival rates. The maximum value for $P_{c o l}$ is 0.0022 , and this achieved at $P_{r k}=0$, where $N=50, t=100, k=5, z=100$, and $\lambda=.2 \mathrm{pac} / \mathrm{s}$.

\section{E. Average Channel Utilization}

Average Channel utilization of the system is calculated according to

$$
\text { Average Channel Utilization }=P_{s} N\left(1-P_{c o l}\right) .
$$

The average channel utilization increases with $N$ as illustrated in Fig. 6. However, since $P_{s}=0.0102$, the average channel utilization in quite low in C-V2X Mode 4. For example, the channel utilization is less than $40 \%$ when $N=1000$.

\section{F. Key Observations}

The average queuing and access delays of a vehicle operating in C-V2X Mode 4 is critical in nature, and need to be minimized for efficient $\mathrm{V} 2 \mathrm{X}$ communication. An interesting observation that we can make through this study is that the average delay can be reduced further by dynamically changing the CAM packet generation rate based on the packet arrival rate of DENM packets. The DENM packet arrival rate is based on the occurrence of an event, and the severity of the event. Based on $\lambda$, if a vehicle can change the CAM packet generation rate to reach the local optimal point of the delay curve shown in Fig. 4, then the vehicle can reduce the overall average delay further in $\mathrm{C}-\mathrm{V} 2 \mathrm{X}$ Mode 4 communication. For example, the average delay considering the curve for $z=100$, $k=9$ and $\lambda=0.2$ pac/s in Fig. 4 can be minimized at $t=300$ ms. Obviously, changing the selection window size from 100 $\mathrm{ms}$ to $50 \mathrm{~ms}$ or $20 \mathrm{~ms}$, as in [5], is another option to reduce the delay associated with $\mathrm{C}-\mathrm{V} 2 \mathrm{X}$ Mode 4 communication.

\section{Conclusions}

This paper has presented a multi-dimensional DTMC model to study the 3GPP C-V2X Mode 4, considering CAM and DENM packets proposed for ITS. The DTMC model that has a dependent node model, a queue model, and two traffic generators has been solved in closed form and iteratively. The performance of a vehicle utilizing the C-V2X Mode 4 is studied using three performance parameters; average delay, collision probability, and average channel utilization. The results has shown that the average delay has a locally optimal combination CAM and DENM packet arrival rate that can be utilized to further reduce delays in the system. The results have also shown low collision probability values thanks to the SPS algorithm, and low average channel utilization. Even the lowest frequency scheduling in Mode 4 is sufficient to cater all possible frequencies of CAM traffic, and hence, it can be concluded that $\mathrm{C}-\mathrm{V} 2 \mathrm{X}$ Mode 4 promises to fulfill the current service requirements of ITS applications conveniently.

\section{ACKNOWLEDGMENT}

This research has been partially financially supported by the ITEA3 project APPSTACLE (15017) and Academy of Finland 6Genesis Flagship (grant 318927).

\section{REFERENCES}

[1] G. Araniti, C. Campolo, M. Condoluci, A. Iera, and A. Molinaro, "LTE for vehicular networking: A survey," IEEE Commun. Mag., vol. 51, no. 5, pp. 148-157, May 2013.

[2] 3GPP TS 36.300, "Evolved universal terrestrial radio access (E- UTRA) and evolved universal terrestrial radio access Network (E- UTRAN); Overall description; Stage 2," Rel-14 V14.1.0, Dec. 2016.

[3] "2 5G Americas V2X cellular solutions." Report, Oct. 2016

[4] 3GPP TS 36.213, "Evolved universal terrestrial radio access (E- UTRA); physical layer procedures," Section 14, Rel-14 V14.2.0, Apr. 2017.

[5] 3GPP TS 36.321, "Evolved universal terrestrial radio access (E- UTRA); Medium access control (MAC) protocol specification," Rel-14 V14.3.0, Jul. 2017.

[6] M. Gonzalez-Martín, M. Sepulcre, R. Molina-Masegosa and J. Gozalvez, "Analytical models of the performance of C-V2X Mode 4 vehicular communications," IEEE Trans. Veh. Technol., vol. 68, no. 2, pp. 11551166, Feb. 2019.

[7] X. Yin, "Performance and reliability evaluation for DSRC vehicular safety communication," Ph.d. Thesis, Dept. Elec. and Com. Eng., Duke University, 2013.

[8] ETSI TS 102 637-2, "Intelligent transport systems (ITS); vehicular communications; basic set of applications; Part 2: specification of cooperative awareness basic service," V1.2.1, Mar. 2011.

[9] J. Santa, F. Pereñíguez, A. Moragón and A. F. Skarmeta, "Vehicleto-infrastructure messaging proposal based on CAM/DENM specifications," IFIP Wireless Days, pp. 1-7, Valencia, Spain, Nov. 2013

[10] D. Martín-Sacristán et al., "Evaluation of LTE-Advanced connectivity options for the provisioning of V2X services," IEEE Wireless Communications and Networking Conference, pp. 1-6, Barcelona, Spain, Apr. 2018. 